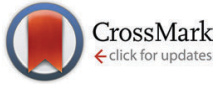

Cite this: Mater. Horiz., 2016, 3, 563

Received 17th July 2016 Accepted 5th September 2016

DOI: $10.1039 / c 6 m h 00250 a$

www.rsc.li/materials-horizons

\section{Sono-assembly of nanostructures via tyrosine- tyrosine coupling reactions at the interface of acoustic cavitation bubbles $\uparrow$}

\author{
Francesca Cavalieri, $\ddagger^{\mathrm{ab}}$ Enrico Colombo, $\ddagger^{\mathrm{c}}$ Eleonora Nicolai, ${ }^{\mathrm{d}}$ Nicola Rosato ${ }^{\mathrm{d}}$ and \\ Muthupandian Ashokkumar*c
}

\begin{abstract}
This work demonstrates that the reactive and oscillating surface of acoustic cavitation microbubbles acts as a catalytic binding site for the coupling of amphiphilic biomolecules bearing tyrosine moieties at the gas-liquid interface. We show a one-step, controlled ultrasonic process to self assemble tyrosine moieties into highly fluorescent uniformly sized sub-micron particles with new optical and functional properties.
\end{abstract}

\section{Introduction}

Phenolic biomolecules have attracted considerable attention in recent years due to the potential use of their oxidized products in medicines, foods, dyes, and cosmetic applications. ${ }^{1}$ In biomedicine, the beneficial effects of polyphenols as antioxidants, antibacterial/anticancer agents ${ }^{2}$ and recently in the treatment of neurodegenerative diseases have been reported. ${ }^{3}$ Phenolic dimers, trimers or oligomers can be generally synthesized by oxidative phenolic coupling processes mediated by inorganic catalysts, UV irradiation $^{4,5}$ and biocatalysts including peroxidases, tyrosinases and laccases. ${ }^{5-7}$ Alternatively, enzyme-free oxidations of phenolic and tyrosine moieties mediated by ${ }^{\bullet} \mathrm{OH}$ radicals have been extensively studied using pulse radiolysis, Fenton-type reactions and low frequency $(20 \mathrm{kHz})$ sonication. ${ }^{8,9}$ However, the oxidation products observed in these free-radical reactions are catechol, hydroquinone, and benzoquinone, whereas the self-assembling of phenolic species has never been observed.

Indeed, high frequency $(0.3-1 \mathrm{MHz})$ ultrasound-induced modifications of phenol-rich biomolecules, including tyrosine

\footnotetext{
${ }^{a}$ Department of Chemical and Biomolecular Engineering,

The University of Melbourne, Parkville, Melbourne, Victoria 3010, Australia

${ }^{b}$ Dipartimento di Scienze e Tecnologie Chimiche, Universita' degli Studi di Roma

"Tor Vergata", via della Ricerca Scientifica 1, 00173, Roma, Italy

${ }^{c}$ School of Chemistry, The University of Melbourne, Parkville, Melbourne,

Victoria 3010, Australia. E-mail: masho@unimelb.edu.au

${ }^{d}$ Department of Experimental Medicine and Surgery University of Rome 'Tor

Vergata', Via Montpellier 1, 00133, Rome, Italy

$\dagger$ Electronic supplementary information (ESI) available: See DOI: 10.1039/c6mh00250a

¥ FC and EC contributed equally to this work.
}

\begin{abstract}
Conceptual insights
Enzyme free oxidations of phenolic and tyrosine moieties mediated by - $\mathrm{OH}$ radicals or molecular oxygen have been extensively studied and have never resulted in the self-assembly of nanoparticles. Here, we emphasize the catalytic role played by the oscillating surface of acoustic cavitation microbubbles in promoting coupling of amphiphilic biomolecules bearing tyrosine moieties at the gas-liquid interface. We demonstrate that the simultaneous presence of ${ }^{\bullet} \mathrm{OH}$ radicals generated by cavitation bubbles at an ultrasonic frequency of $355 \mathrm{kHz}$, hot bubble surface and surface-active phenolic species results in unexpected coupling reactions and nanoparticle formation. Nanoparticles possess remarkable optical properties emitting blue, green and red light because of the chemical coupling reactions and $\pi-\pi$ stacking interactions between oligomers. Additionally, unlike the starting tyrosine moiety, these newly synthesized phenolic nanoparticles are endowed with a significant antioxidant property. This is a new conceptual insight into the use of high frequency ultrasound to assemble phenolic building blocks into complex nanostructures possessing new functional properties.
\end{abstract}

and proteins, have never been investigated. These studies are relevant for understanding possible in vivo reactions induced by ultrasound used in medicines for therapeutic purposes. In fact, High Intensity Focused Ultrasound (HIFU) for ablation of tumors $^{10}$ and acoustic targeted drug delivery in brain tissues ${ }^{11}$ require significantly high acoustic power levels and therefore the occurrence of significant cavitation activity is possible. Studies on the ultrasonic reactivity of phenolic biomolecules also pave the way for the development of a new methodology to assemble phenolic building blocks into complex nanostructures. The currently available approaches for the manipulation of phenol moieties into the desired nano-architectures require the formation of catechol-metal coordination complexes or oxidative coupling reactions catalyzed by metals or enzymes. For instance, metal-polyphenol thin films and microcapsules were recently engineered exploiting the coordination of tannic acid and $\mathrm{Fe}(\mathrm{III})$ ions. ${ }^{12-14}$ Green luminescent, porous and hollow microspheres were prepared via $\mathrm{Cu}^{2+}$ and temperature mediated oxidative coupling assembly of green tea polyphenols in water. $^{13}$ 
Here, we demonstrate that tyrosine derivatives can undergo a number of oxidative modifications upon high frequency $(\sim 0.35-1 \mathrm{MHz})$ ultrasound treatment to form hydroxylated tyrosines, dimers and oligomers. The oligomers self-assemble into fluorescent, monodisperse and functional nanoparticles without the assistance of metal coordination. We employed the same method to crosslink tyrosine-bearing proteins leading to the formation of protein nanoparticles. For the first time, we emphasize the catalytic role of reactive surfaces of cavitation bubbles and we introduce a new strategy to fabricate nanoparticles exploiting tyrosine-tyrosine coupling without resorting to metals or enzymes.

To investigate ultrasound assisted oxidization and assembly of tyrosine moieties, $N$-benzoyl---tyrosine ethyl ester (BTTE) was utilized as a model system. BTEE was chosen because of its hydrophobic properties to maximize its diffusion to the gas bubble/liquid interface. In addition, BTTE mimics the hydrophobic environment experienced by tyrosine within a protein. An aqueous solution of $1 \mathrm{mM}$ BTTE was sonicated under an air atmosphere, at $37{ }^{\circ} \mathrm{C}$ and delivered acoustic power of $1.83 \mathrm{~W} \mathrm{~cm}^{-2}$, up to 3 hours at $355 \mathrm{kHz}$ and $1 \mathrm{MHz}$ at $\mathrm{pH} 4$ to prevent the possible oxidation of quinones by air, which generally requires alkaline conditions. ${ }^{15}$ Under the experimental conditions used in this study, the thermal decomposition of water vapor within imploding cavitation bubbles generates ${ }^{\circ} \mathrm{OH}$ and ${ }^{\bullet} \mathrm{H}$ primary radicals, which, on further reaction, leads to the formation of hydrogen peroxide and superoxide. ${ }^{16}$ When surface-active and non-volatile solutes or macromolecules are dissolved in the aqueous medium, sonochemical reactions can occur at the bubble-solution interface and in bulk solution. ${ }^{17,18}$

The conversion of BTTE into the oxidation products upon ultrasonic treatment was monitored by HPLC analysis, mass spectrometry (MS) and fluorescence spectroscopy. The HPLC chromatograms and MS analysis results of the oxidation products after sonication are shown in Fig. 1.

These results indicate a complete conversion of BTTE into BTTE hydroxylated species and higher molecular weight dimers and oligomers (Fig. 1a and b).

In particular, the mass spectra of oxidation products (Fig. 1c) obtained after $0.5 \mathrm{~h}$ of sonication indicated the formation of OH-BTEE at $330 \mathrm{~m} / \mathrm{z}$, OH-BTEE/BTTE dimers at $665 \mathrm{~m} / \mathrm{z}$ and OH-BTEE/OH-BTTE dimers at $681 \mathrm{~m} / z$. Interestingly, nanoparticles are formed after $1 \mathrm{~h}$ of sonication, indicating that the sequential coupling of dimers leads to oligomers able to selfassemble. The colloidal nanoparticles (yield $40 \%$ ) were collected by centrifugation, washed, dissolved in acetonitrile and analyzed by MS (Fig. 1d) to determine their composition. Higher molecular weight BTTE oligomers were identified by the multiple peaks in the range of $850-1600 \mathrm{~m} / \mathrm{z}$ (Fig. 1d) corresponding approximately to 4-5 OH-BTTE repeating units. We also observed the nanoparticle formation at the two acoustic power levels of $1.83 \mathrm{~W} \mathrm{~cm}^{-2}$ and $0.9 \mathrm{~W} \mathrm{~cm}^{-2}$ after $1 \mathrm{~h}$ of sonication.

Further insight into the BTTE oxidation and coupling reactions induced by the ultrasonic treatment was obtained using fluorescence spectroscopy. The fluorescence spectra of the oxidation products measured in PBS $\mathrm{pH} 7.4$ as a function of sonication time

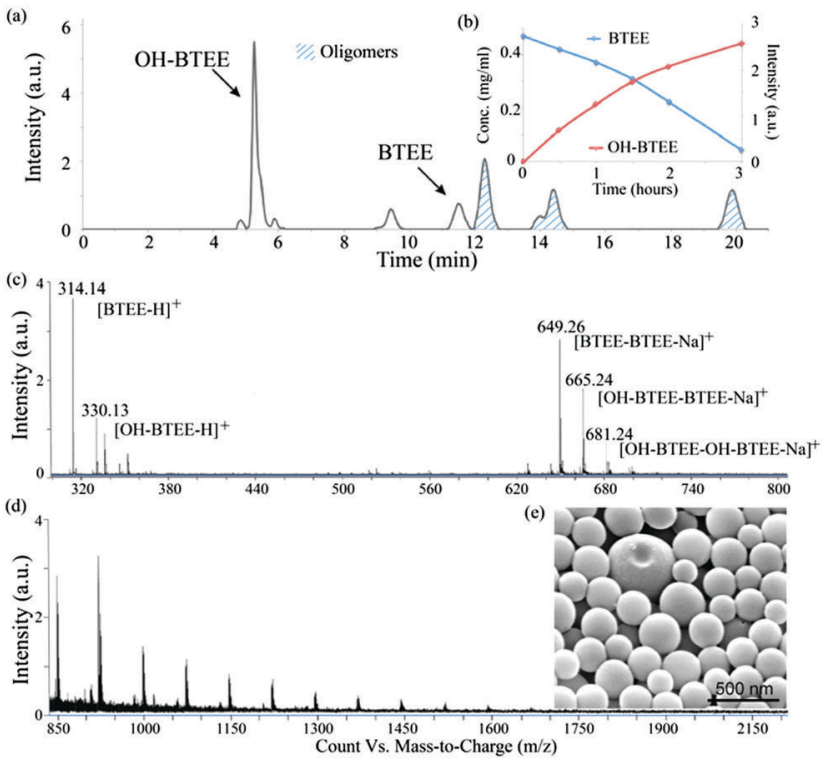

Fig. 1 The oxidation of BTTE was monitored using HPLC and MS; (a) HPLC analysis of BTEE sonicated at $355 \mathrm{kHz}$ for 30 minutes; (b) the intensities of the BTTE peak at $11.5 \mathrm{~min}$ and $\mathrm{OH}$-BTEE at $5.3 \mathrm{~min}$ are plotted as a function of sonication time; (c) mass spectra of BTTE oxidation products after 30 minutes of sonication; (d) MS of oligomer nanoparticles produced after three hours of sonication and dissolved in acetonitrile; and (e) SEM images of nanoparticles obtained during sonication.

(Fig. 2a) show a peak centred at $420 \mathrm{~nm}$, which increases in intensity and is red-shifted up to $446 \mathrm{~nm}$ at longer sonication times. These results indicate that a number of different fluorescent species were formed during the ultrasonic treatment of BTTE. Catecholic compounds and o-quinones do not exhibit fluorescence in this region. Dityrosine and related phenolic dimers exhibit fluorescence in the $400-500 \mathrm{~nm}$ spectral range, upon excitation within 320 and $380 \mathrm{~nm}$, originating from the conjugated system and the phenolate form of the excited single state. $^{19}$

In our study, the intense fluorescence emission observed at neutral pH (Fig. 2a) is consistent with the conversion of BTTE into dimeric and oligomeric forms, as identified by MS.

The presence of different fluorescent species contributing to the overall fluorescence is also confirmed by the red shift observed in the emission peak, while changing the excitation wavelength (Fig. S1a, ESI $\dagger$ ), and by the excitation spectra (Fig. S1b, ESI $\dagger$ ). We observed that the enhancement in fluorescence continues with sonication and levels off when sonication is ceased, suggesting that the acoustic cavitation generated ${ }^{\bullet} \mathrm{OH}$ radicals are responsible for the enhancement in fluorescence rather than spontaneous oxidation. It is worth mentioning that the high temperature reached within the cavitation bubble causes the dissociation of $\mathrm{N}_{2}$ and $\mathrm{O}_{2}$ present in air. The dissociated species may form $\mathrm{NO}_{x}, \mathrm{HNO}_{2}$ and $\mathrm{HNO}_{3}{ }^{20}$ Although a possible role of $\mathrm{NO}_{x}$ species in the oxidation of BTTE cannot be ruled out, the concentration of these species is very low compared to - $\mathrm{OH}$ radicals. Previous studies have shown that oxidation by ${ }^{\circ} \mathrm{OH}$ radicals is the most dominant mechanism. 


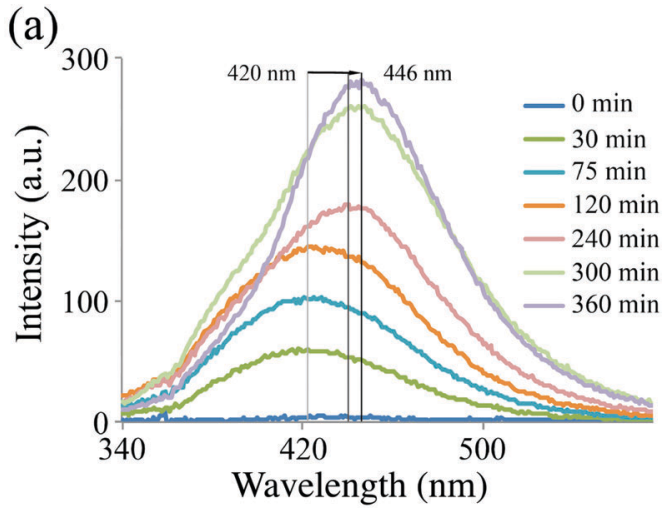

(b)

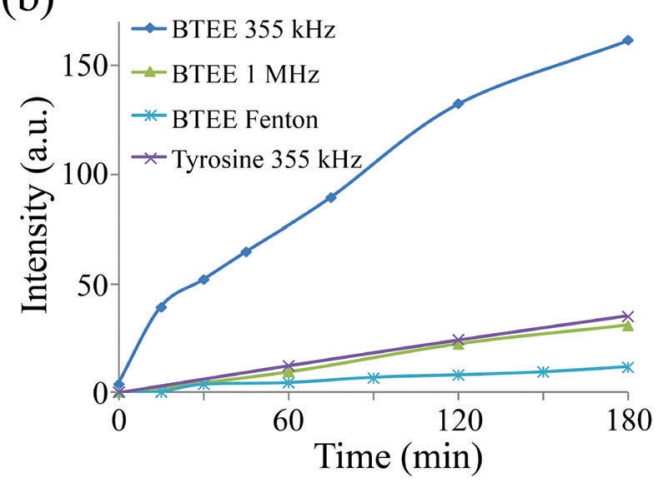

Fig. 2 The fluorescence emission of BTTE oxidation products. (a) Fluorescence emission spectra $\left(\lambda_{\text {ex }} 320 \mathrm{~nm}\right.$ ) of the BTTE dimerization products formed during sonication ( $355 \mathrm{KHz}, 1.83 \mathrm{~W} \mathrm{~cm}^{-2}$ ) and (b) temporal evolution of emission intensity arising from samples sonicated using different ultrasonic frequencies. Fluorescence emission of BTTE treated using the radicals generated by the Fenton reaction is shown for comparison.

To ascertain the role of acoustic cavitation in promoting BTTE coupling reactions, a comparison of fluorescent species formed under different experimental conditions was performed. Fig. $2 \mathrm{~b}$ shows that the generation of ${ }^{\bullet} \mathrm{OH}$ radicals in the same concentration range $\left(240 \mu \mathrm{M} \mathrm{h}^{-1}\right)$ by Fenton's reagents did not result in the formation of fluorescent BTTE dimers. This suggests that the simultaneous presence of ${ }^{\bullet} \mathrm{OH}$ radicals and a reactive cavitation bubble interface are necessary for the coupling of BTTE molecules.

Next, the reactivities of BTTE and tyrosine were compared to investigate the relationship between the coupling reaction and the hydrophilicity of the phenolic moiety. Compared to BTTE, the sonication of the more hydrophilic tyrosine resulted in a modest fluorescence increase, indicating a lack of dimer formation. In agreement with this, the HPLC analysis of the sonicated tyrosine solution showed mainly the presence of hydroxylated products (2.7 $\mathrm{min}$ ) and only a weak peak (6 $\mathrm{min}$ ) ascribed to dimers (Fig. S2, ESI $\dagger$ ). These results are also consistent with previous studies on ${ }^{\bullet} \mathrm{OH}$ radical mediated tyrosine oxidation via pulse radiolysis or Fenton-type reactions. ${ }^{8,9}$ The ultrasonic oxidation of more hydrophilic phenolic molecules such as tyrosine seems to occur in the bulk phase rather than at the interface of the cavitation bubbles. Taken together, these comparative studies reveal that the combination of ${ }^{\bullet} \mathrm{OH}$ radicals generated by reactive cavitation bubbles and surface-active phenolic species results in unexpected coupling reactions.

To gain more insight into the role of the oscillating bubbles in determining the coupling reactions, the effect of frequency was explored. Experiments were carried out at $20 \mathrm{kHz}$ and $1 \mathrm{MHz}$. We observed only 10\% BTTE dimerization when $20 \mathrm{kHz}$ sonication was used (data not shown). This suggests that the low frequency sonication of BTTE is ineffective, likely due to a low amount of ${ }^{\bullet} \mathrm{OH}$ radicals generated at $20 \mathrm{kHz}$. We observed that $355 \mathrm{kHz}$ is the optimal frequency, in the frequency range investigated, generating sufficient amount of ${ }^{\bullet} \mathrm{OH}$ radicals simultaneously providing the required interfacial temperature. It is known that an increase in the ultrasound frequency from $355 \mathrm{kHz}$ to $1 \mathrm{MHz}$ results in a 3 -fold decrease in ${ }^{\circ} \mathrm{OH}$ yield. For instance after 1 hour of sonication $120 \mu \mathrm{M}$ and $40 \mu \mathrm{M} \cdot \mathrm{OH}$ radicals were generated at $355 \mathrm{kHz}$ and $1 \mathrm{MHz}$, respectively. ${ }^{20}$ This decrease is due to the fact that at higher frequencies the acoustic cycle becomes very short, which restricts the amount of water vapor that can evaporate into the bubble during the expansion phase of the acoustic cycle. A decrease in the amount of water vapour within the collapsing bubble leads to a decrease in the amount of ${ }^{\bullet} \mathrm{OH}$ radicals generated. ${ }^{21} \mathrm{~A}$ significant reduction in dimer formation (Fig. 2b) was observed during the ultrasonic treatment of BTTE at the same power but at a higher frequency (1 MHz). However, Fig. 2b shows that the yield of fluorescent species generated at $1 \mathrm{MHz}$ is much lower than 3-fold, indicating that an additional factor contributes in reducing the reactivity of bubbles oscillating at higher frequencies. The catalytic coupling of tyrosyl radicals at the bubble-solution interface implies a mechanism (Scheme 1) whereby the aromatic rings of the BTTE molecules should approach each other prior to their oxidation and coupling. As shown in Scheme 1, we speculate that BTTE and OH-BTTE molecules adsorb at the air-liquid interface of the cavitation bubbles. It was found that chemically active cavitation bubbles exist for about $0.3-0.1 \mathrm{~ms}$, in the ultrasound frequency range of $200 \mathrm{kHz}$ to $1 \mathrm{MHz}{ }^{18,22}$ The cavitation bubbles referred to here are stable (repetitive transient) bubbles. $^{23}$ This is a sufficient time, based on the diffusion coefficients measured for different amino acids, which span from 7 to $10 \times 10^{-10} \mathrm{~m}^{2} \mathrm{~s}^{-1}$ for BTTE to diffuse, adsorb and react at the bubble-solution interface, as shown in Scheme 1. However, a faster oscillation of cavitation bubbles produced at $1 \mathrm{MHz}$ before collapse is less effective in collecting BTTE molecules at the air-liquid interface. Higher shear forces and temperature in the proximity of the cavitation bubbles generated at $0.35 \mathrm{MHz}$, compared to $1 \mathrm{MHz}$, may also contribute to accelerate the coupling and oligomerization reactions, shown in Scheme 1. The above discussion supports the fact that acoustic cavitation bubbles play a catalytic role in inducing the oligomerization of BTTE molecules at the liquid-gas interface, which is pictorially shown in Scheme 1.

Interestingly, insoluble BTTE oligomers self-assemble into organized nanoparticles (see Scheme 1) during sonication performed under either air or a depleted oxygen atmosphere by nitrogen bubbling. This suggests that the superoxides, generated by ultrasound in the presence of oxygen, are not involved in the 


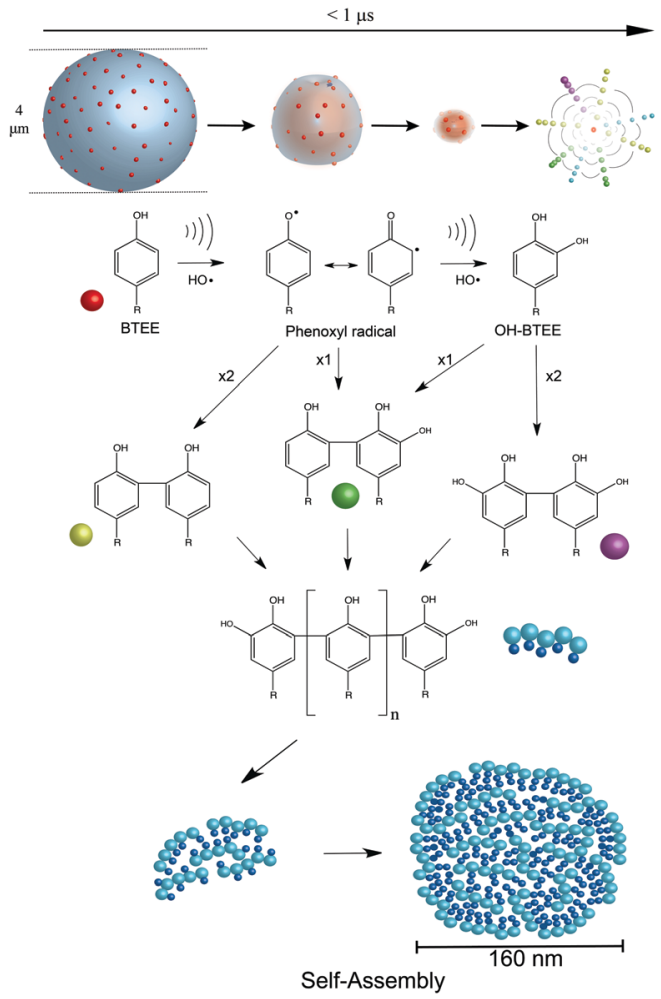

Scheme 1 Reaction scheme for the ultrasonic coupling of tyrosine moieties into hydroxylated species and oligomers to form nanoparticles by selfassembly. The time scale provided for the bubble collapse $(<1 \mu \mathrm{s})$ is based on single bubble dynamics calculations. ${ }^{23}$ This process can repeat over many acoustic cycles due to stable cavitation as mentioned in the discussion.

process and confirms the role played by ${ }^{\bullet} \mathrm{OH}$ radicals in BTTE oxidation and coupling reactions. The uniform spherical nanoparticles, formed under air (Fig. 3a) and nitrogen (Fig. 1e) atmospheres, were imaged and analyzed using SEM.

The average diameter of the nanoparticles was approximately $220 \pm 50 \mathrm{~nm}$, and $\zeta$ potential measurements indicated that the nanoparticle surface was negatively charged $(-20 \pm 5 \mathrm{mV})$. It is known that tyrosine oligomers have a phenolic $\mathrm{p} K_{\mathrm{a}}$ near $7^{19}$ and hence BTTE oligomers are present in the ionized form conferring a negatively charged surface to the nanoparticles, consequently providing colloidal stability.

Nanoparticles disassemble completely in methanol or in alkaline aqueous solution ( $\mathrm{pH} 10)$. This indicates that the $\pi-\pi$ stacking interactions between the aromatic moieties are likely the driving force for BTTE oligomer assembly. The organic solvent and the electrostatic repulsion between phenolate ions induce the dissolution of nanoparticles into soluble oligomers.

To confirm this hypothesis, the optical properties of the nanoparticles were investigated and compared to fluorescence properties of the soluble BTTE dimers and trimers. In addition to the already observed fluorescence emission in the range of 340-500 nm (Fig. 3b), the emission spectrum of the nanoparticles revealed a new band in the range of 500-700 $\mathrm{nm}$ centred at $510 \mathrm{~nm}$ (Fig. 3c), when excited at $480 \mathrm{~nm}$. The corresponding excitation spectrum showed a peak centred at $490 \mathrm{~nm}$ (Fig. 3c).

Fluorescence microscopy images confirmed that the nanoparticles generate blue, green and red emissions (Fig. 3a). The 500-700 $\mathrm{nm}$ emission band disappears upon dissolution of nanoparticles in alkaline solution (Fig. 3d), whereas the fluorescence properties of the soluble oligomers were resumed.

This suggests that the $\pi-\pi$ stacking interactions between oligomers in the nanoparticles reduce the excited state energy resulting in the additional red shifted emission band at $510 \mathrm{~nm}$. The spectroscopic properties exhibited by the BTTE nanoparticles evoke the fluorescence properties of GPF and its mutant YFP. In these proteins the green light emission of conjugated tyrosines is red shifted by the $\pi-\pi$ stacking interactions between tyrosine and the phenolate anion. ${ }^{24}$ In addition, the nanoparticles exhibited a good photostability after continuous irradiation for 10 min under photoirradiation (Fig. S3, ESI $\dagger$ ).

We next evaluated the antioxidant properties of BTTE nanoparticles after dissolution, using the assay based on DPPH radical reduction. Ascorbic acid was used as a comparative antioxidant system to evaluate the antioxidant activity of BTTE oligomers (Table 1).
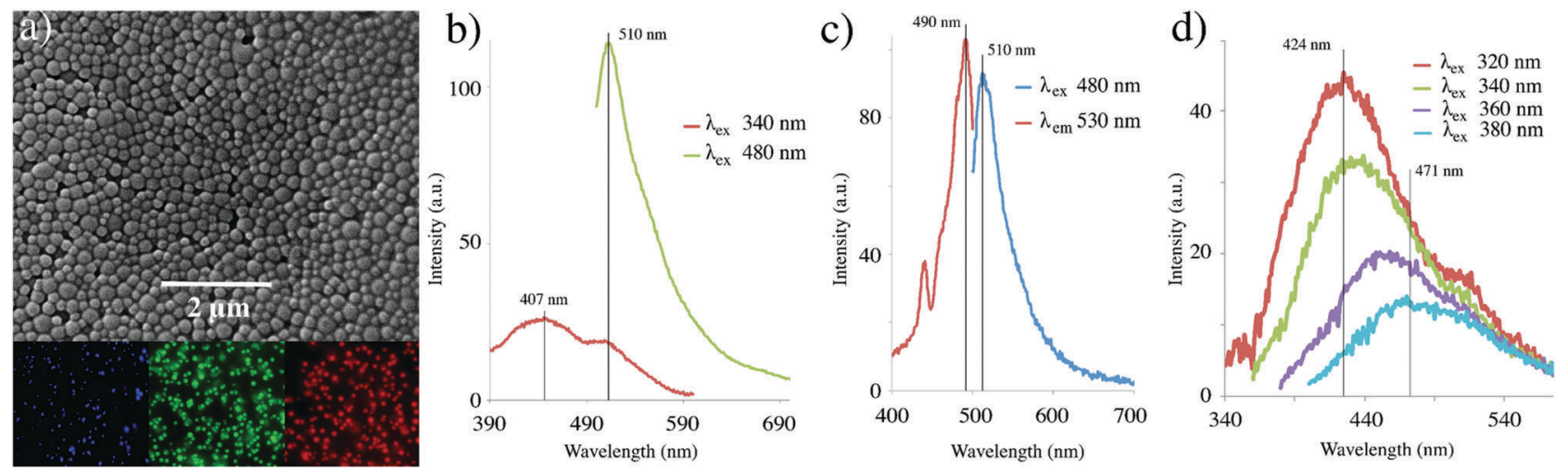

Fig. 3 The microscopy and fluorescence properties of oligomeric nanoparticles; (a) SEM and fluorescence microscopy images of BTTE nanoparticles showing blue, green and red emission. (b) Fluorescence emission spectra of BTTE oligomeric nanoparticles excited at $480 \mathrm{~nm}$ (green line) and $320 \mathrm{~nm}$ (red line), (c) excitation (red line) and emission (light blue) spectra of BTTE oligomeric nanoparticles, and (d) fluorescence emission spectra of BTTE oligomeric nanoparticles after dissolution in alkaline solution. 
Table 1 Antioxidant activity of ascorbic acid (taken as 100\%), BTEE NPs and BTEE

\begin{tabular}{lc}
\hline Compound & Antioxidant activity (relative \%) \\
\hline Ascorbic acid & 100 \\
BTEE NPs & 56 \\
BTEE not treated & 3 \\
Blank & 0
\end{tabular}

At the same concentration, untreated BTTE possesses a negligible radical scavenging ability, whereas BTTE oligomers significantly quenched the DPPH radicals.

We have ascribed the improved antioxidant properties of the BTTE oligomers to the extensive hydroxylation of conjugated rings. It is known that the position and the degree of hydroxylation are of primary importance in determining the antioxidant activity of natural polyphenols. ${ }^{25}$

Finally, our findings (Fig. S3 and S4, ESI $\dagger$ ) reveal that under ultrasonic treatment, tyrosine moieties embedded in the protein chains can combine to form either intramolecular or intermolecular blue and red fluorescent dimers and eventually promote nanoparticle (150 $\mathrm{nm}$ in diameter) formation.

\section{Conclusions}

In conclusion, we have shown that the reactive and oscillating surface of cavitation microbubbles acts as a catalytic binding site to dimerize and polymerize amphiphilic biomolecules bearing tyrosine moieties at the gas-liquid interface. The ultrasonic oxidation of tyrosine bearing biomolecules results in the formation of highly fluorescent and antioxidant nanoparticles. We propose a single-step controlled ultrasonic approach as a strategy for the fabrication of nanoparticles without using external oxidants, inorganic catalysts or enzymes, thus avoiding complicated preparation steps and potential toxic agents. Fluorescent tyrosine- and protein-based nanoparticles simply prepared using high frequency ultrasound are promising materials for use as antioxidant and imaging agents.

\section{Acknowledgements}

This research was funded by the ARC Future Fellowship 2014 (project number FT140100873, to FC). EC acknowledges the University of Melbourne for the scholarships (MIRS/MIFRS).

\section{Notes and references}

1 (a) J. G. Handique and J. B. Baruah, React. Funct. Polym., 2002, 52, 163-188; (b) A. Heleno, A. Martins, M. J. Queiroz and I. C. Ferreira, Food Chem., 2015, 173, 501-513; (c) T. Jähnert, M. D. Hager and U. S. Schubert, J. Mater. Chem. A, 2014, 2, 15234-15251; (d) O. V. Zillich, U. Schweiggert-Weisz, P. Eisner and M. Kerscher, Int. J. Cosmet. Sci., 2015, 37, 455-464.

2 (a) N. T. Zaveri, Life Sci., 2006, 78, 2073-2080; (b) M. Daglia, Curr. Opin. Biotechnol., 2012, 23, 174-181.
3 C. Ramassamy, Eur. J. Pharmacol., 2006, 545, 51-64.

4 C. Houee-Levin, K. Bobrowski, L. Horakova, B. Karademir, C. Schoneich, M. J. Davies and C. M. Spickett, Free Radical Res., 2015, 49, 347-373.

5 (a) T. Michon, M. Chenu, N. Kellershon, M. Desmadril and J. Guéguen, Biochemistry, 1997, 36, 8504-8513; (b) D. Šmejkalová, P. Conte and A. Piccolo, Biomacromolecules, 2007, 8, 737-743; (c) M. Wilchek and T. Miron, Bioconjugate Chem., 2015, 26, 502-510; (d) H. Eickhoff, G. Jung and A. Rieker, Tetrahedron, 2001, 57, 353-364.

6 T. S. Wang, M. C. Wang and P. M. Huang, Soil Sci., 1983, 136, 226-230.

7 T. Heck, G. Faccio, M. Richter and L. Thony-Meyer, Appl. Microbiol. Biotechnol., 2013, 97, 461-475.

8 (a) T. Heck, G. Faccio, M. Richter and L. Thöny-Meyer, Appl. Microbiol. Biotechnol., 2013, 97, 461-475; (b) G. Cohen, S. Yakushin and D. Dembiec-Cohen, Anal. Biochem., 1998, 263, 232-239.

9 N. Serpone, R. Terzian, P. Colarusso, C. Minero, E. Pelizzetti and H. Hidaka, Res. Chem. Intermed., 1992, 18, 183-202.

10 Y. F Zhou, World J. Clin. Oncol., 2011, 2, 8-27.

11 A. Burgess and K. Hynynen, ACS Chem. Neurosci., 2013, 4, 519-526.

12 H. Ejima, J. J. Richardson, K. Liang, J. P. Best, M. P. van Koeverden, G. K. Such, J. Cui and F. Caruso, Science, 2013, 341, 154-157.

13 Z. Chen, C. Wang, J. Chen and X. Li, J. Am. Chem. Soc., 2013, 135, 4179-4182.

14 J. Fei, J. Zhao, C. Du, A. Wang, H. Zhang, L. Dai and J. Li, ACS Nano, 2014, 8, 8529-8536.

15 S. I. Sadykh-Zade, A. V. Ragimov, S. Suleimanova and V. I. Liogon'Kii, Polym. Sci., 1972, 14, 1395-1403.

16 K. S. Suslick, J. Acoust. Soc. Am., 1991, 89, 1885-1886.

17 S. Manickam and M. Ashokkumar, Cavitation: A Novel Energy-efficient Technique for the Generation of Nanomaterials, CRC Press, 2014.

18 L. Dharmarathne, M. Ashokkumar and F. Grieser, J. Phys. Chem. A, 2012, 116, 7775-7782.

19 (a) D. A. Malencik, J. F. Sprouse, C. A. Swanson and S. R. Anderson, Anal. Biochem., 1996, 242, 202-213; (b) G. J. Smith and T. G. Haskell, J. Photochem. Photobiol., B, 2000, 55, 103-108.

20 T. Ouerhani, R. Pflieger, W. Ben Messaoud and S. I. Nikitenko, J. Phys. Chem. B, 2015, 119, 15885.

21 M. Ashokkumar, D. Sunartio, S. Kentish, R. Mawson, L. Simons, K. Vilkhu and C. Versteeg, Innovative Food Sci. Emerging Technol., 2008, 9, 155-160.

22 D. Sunartio, M. Ashokkumar and F. Grieser, J. Am. Chem. Soc., 2007, 129, 6031-6036.

23 T. Leighton, The Acoustic Bubble, Academic Press, 1994.

24 R. Y. Tsien, Annu. Rev. Biochem., 1998, 67, 509-544.

25 D. E. Pratt, Phenolic Compounds in Food and Their Effects on Health II ACS Symposium Series, 1992, vol. 507, pp. 54-71. 